\title{
Mitogen-Activated Protein Kinase/Extracellular Signal-Regulated Kinase Activation in Somatodendritic Compartments: Roles of Action Potentials, Frequency, and Mode of Calcium Entry
}

\author{
Serena M. Dudek and R. Douglas Fields \\ National Institute of Child Health and Human Development, National Institutes of Health, Bethesda, Maryland 20892
}

Mitogen-activated protein kinase (MAPK) has been identified as a potential element in regulating excitability, long-term potentiation (LTP), and gene expression in hippocampal neurons. The objective of the present study was to determine whether the pattern and intensity of synaptic activity could differentially regulate MAPK phosphorylation via selective activation of different modes of calcium influx into CA1 pyramidal neurons. An antibody specific for the phosphorylated (active) form of MAPK was used to stain sections from hippocampal slices, which were first stimulated in vitro.

LTP-inducing stimulation [theta-burst (TBS) and $100 \mathrm{~Hz}$ ] was effective in inducing intense staining in both dendritic and somatic compartments of CA1 neurons. Phosphorylation of MAPK was also induced, however, with stimulation frequencies (3-10 Hz) not typically effective in inducing LTP. Intensity and extent of staining was better correlated with the spread of

A defining feature of long-term potentiation (LTP) is its dependence on certain patterns of afferent stimulation. LTP is typically induced by trains of high-frequency stimulation $(\geq 100 \mathrm{~Hz})$ (Bliss and Lomo, 1973) or by brief, high-frequency bursts repeated at the theta rhythm (Larson et al., 1986); lower frequencies of stimulation $(1-20 \mathrm{~Hz})$ can result in short-term increases in synaptic strength [short-term potentiation (STP)] (Cummings et al., 1996) or decreases in synaptic strength [long-term depression (LTD)] (Dudek and Bear, 1992) (but see Thomas et al., 1998). On a molecular level, stimulus frequency-dependent differences in the induction of LTP, STP, and LTD have been associated with the concentration of intracellular calcium induced by the associated stimulus frequencies (Cummings et al., 1996), but the subsequent calcium-dependent reactions are only beginning to be identified and investigated. Related to the induction of the latephase of LTP are experiments in culture showing that the mode of calcium entry, i.e., synaptic, through NMDA receptors or somatic, through voltage sensitive calcium channels (VSCCs) can determine which calcium-dependent signaling cascades and subsequent gene expressions are activated (for review, see Bading et al., 1993; Diesseroth et al., 1996; Ginty, 1997). Because firing frequency and the amplitude of the synaptic potential (propor-

\footnotetext{
Received Sept. 20, 2000; revised Oct. 26, 2000; accepted Nov. 3, 2000.

We thank Taryn Hogan for acquiring images, Dr. Annadora Bruce-Keller for her valuable technical suggestions, and our many colleagues who took their time to provide comments on this manuscript.

Correspondence should be addressed to R. Douglas Fields, National Institute of Child Health and Human Development, National Institutes of Health, Building 49, Room 5A38, MSC 4480, 49 Convent Drive, Bethesda, MD 20892. E-mail: fields@helix.nih.gov.

Copyright $(\subset 2001$ Society for Neuroscience $0270-6474 / 01 / 210001-\bullet \$ 15.00 / 0$
}

population spikes across the CA1 subfield than with frequency (above $3 \mathrm{~Hz}$ ). Experiments using inhibitors of NMDA receptors and voltage-sensitive calcium channels (VSCCs) revealed that, although MAPK is activated after both TBS and $5 \mathrm{~Hz}$ stimulation, the relative contribution of calcium through L-type calcium channels differs. Blockade of NMDA receptors alone was sufficient to prevent MAPK phosphorylation in response to $5 \mathrm{~Hz}$ stimulation, whereas inhibitors of both NMDA receptors and VSCCs were necessary for inhibition of the TBS-induced staining. We conclude that the intensity and frequency of synaptic input to CA1 hippocampal neurons are critically involved in determining the path by which second-messenger cascades are activated to activate MAPK.

Key words: long-term potentiation; hippocampus; ERK; activity-dependent; dendrite; soma; CA1 tional to stimulus intensity) are important factors in regulating dendritic and nuclear calcium dynamics (Nakazawa and Murphy, 1999), it is likely that they could similarly mediate plasticity and signaling to the nucleus.

Mitogen-activated protein kinases (MAPK), particularly the extracellular signal regulated kinase (ERKI/II), can respond to stimulation by neurotrophins, neuromodulators, and intracellular calcium and can lead to the phosphorylation of cAMP response element-binding protein (CREB) and other transcription factors to mediate gene expression (for review, see Rosen et al., 1994; Xia et al., 1996; Impey et al., 1999). In addition, activation of MAPK has also been proposed to regulate LTP in CA1 of the hippocampus. Contributing to this view are numerous studies showing the following: (1) inhibitors of MAPK block LTP induction and/or its late-phase expression (English and Sweatt, 1997; Impey et al., 1998) (but see Liu et al., 1999; Winder et al., 1999; Kanterewicz et al., 2000); (2) glutamate and potassium are very

This article is published in The Journal of Neuroscience, Rapid Communications Section, which publishes brief, peerreviewed papers online, not in print. Rapid Communications are posted online approximately one month earlier than they would appear if printed. They are listed in the Table of Contents of the next open issue of JNeurosci. Cite this article as: JNeurosci, 2001, 21:RC122 (1-5). The publication date is the date of posting online at www.jneurosci.org.

http://www.jneurosci.org/cgi/content/full/4915 
effective in activating MAPK in cultured hippocampal neurons and in slices (Bading and Greenberg, 1991; Kurino et al., 1995; Baron et al., 1996); and (3) MAPK is activated both in slices and in vivo by LTP-inducing stimulation and certain learning paradigms (English and Sweatt, 1996; Atkins et al., 1998; Blum et al., 1999; Davis et al., 2000). Furthermore, because MAPK is localized in both cell bodies and dendrites of neurons (Fiore et al., 1993), it may also play a role in modulating cellular and dendritic excitability (Winder et al., 1999) or cytoskeletal function (Quinlan and Halpain, 1996). The potential for subcellular heterogeneity of MAPK activation via different types of stimuli, therefore, could be an important factor in initiating distinct forms of plasticity by different patterns of activation. In this study, we examined the dependence of MAPK phosphorylation on the frequency and intensity of synaptic activity and whether different modes of calcium entry (mediated via NMDA receptors vs VSCCs) or signaling pathways in spatially distinct cellular compartments contribute differentially to activate MAPK in CA1 neurons.

\section{MATERIALS AND METHODS}

Slice preparation and physiology. Hippocampal slices $(400 \mu \mathrm{m})$ were prepared from hooded or albino Sprague Dawley rats between the ages of 5 and 12 weeks, although similar results were obtained with tissue from mice of different strains. Slices were cut on a vibraslicer in ice-cold artificial CSF (ACSF) containing (in mM): $\mathrm{NaCl} 124, \mathrm{KCl} 4, \mathrm{NaH}_{2} \mathrm{PO}_{4}$ $1.25, \mathrm{NaHCO}_{2} 26, \mathrm{CaCl}_{2} 2, \mathrm{MgCl}_{2} 2$, and glucose 10 , bubbled with $95 \%$ $\mathrm{O}_{2}-5 \% \mathrm{CO}_{2}$. Slices were perfused at $1 \mathrm{ml} / \mathrm{min}$ in an interface chamber [Medical Systems/Haas (Greenvale, NY) top; larger synaptic potentials were observed using the Oslo/Fine Science Tools (Foster City, CA)-type interface chamber, but the design of the Haas chamber was better-suited for quick removal of slices with minimal mechanical manipulation]. Slices were maintained at $34^{\circ} \mathrm{C}$ because comparatively little staining for phospho-MAPK could be induced when slices were incubated at $28^{\circ} \mathrm{C}$. Concentric bipolar stimulating electrodes (Frederick Haer \& Co. Inc., Brunswick, ME) were placed in the stratum radiatum, and for recording of population-spikes, an ACSF-containing glass recording electrode was placed in stratum pyramidale. Unless stated otherwise, stimulation was delivered with an intensity of $140 \mu \mathrm{A}$ and a duration of $50 \mu \mathrm{sec}$. In all experiments, slices were stimulated with 120 pulses, because fewer than 80 pulses did not reliably produce staining. Theta-burst stimulation (TBS) (Larson et al., 1986) consisted of 10 bursts of four pulses at 100 $\mathrm{Hz}$, delivered at $5 \mathrm{~Hz}$, given a total of three times, with a 15-30 sec interval. Because MAPK is rapidly dephosphorylated, slices were fixed in 4\% paraformaldehyde 1-3 min after the last pulse of stimulation (less staining, in a perinuclear pattern, was observed when slices were fixed within $60 \mathrm{sec}$ of stimulation); no attempt was made to determine whether LTP had been induced in each slice because of the requirement for rapid fixation. Tests of LTP induction were often used in other slices to determine wash-in of drugs, such as APV. Drugs were purchased from Tocris Cookson (Ballwin, MO).

Immunocytochemistry. Slices were fixed overnight and then cryoprotected with a $24 \mathrm{hr}$ incubation in $10 \%$ sucrose-4\% paraformaldehyde. Frozen slices were recut at $30 \mu \mathrm{m}$, and the resulting sections washed three times in Tris-buffered saline, incubated for $30 \mathrm{~min}$ in $50 \%$ ethanol, permeabilized with three 15 min washes in $0.3 \%$ Triton $\mathrm{X}-100$ in $1 \%$ normal goat serum (NGS), and blocked for $1 \mathrm{hr}$ with $3 \% \mathrm{NGS}, 0.3 \%$ Triton X-100. Primary antibody to dually phosphorylated ERK I/II (Promega, Madison, WI) in 1\% NGS was used at a 1:7000-1:10,000 dilution for $48 \mathrm{hr}$. Sections were washed, incubated with a biotinylated secondary antibody at 1:1000 for $90 \mathrm{~min}$, washed again, incubated for 45 min with avidin-biotin complex (Vector Elite ABC; Vector Laboratories, Burlingame, CA), and then processed for DAB reaction product. To avoid possible saturation of the $\mathrm{DAB}$ reaction product, the antibody concentration was chosen to be sufficiently dilute to allow the reaction to proceed at a rate slow enough to permit cessation of the reaction before the point of maximum intensity of stain. The reaction product was specific for a MAPK- kinase (MEK)-dependent process because staining was essentially eliminated by $20 \mu \mathrm{M}$ of the MEK1 and MEK 2 inhibitor U0126 (data not shown).

Images $(4$ or $20 \times$ ) were acquired digitally on a microscope equipped with a SPOT digital camera (Diagnostic Instruments, Sterling Heights,
MI), and subsequently analyzed with Metamorph software (Universal Imaging, West Chester, PA). The three most representative and/or most complete sections from each slice were chosen for analysis, and the average intensity was determined in $20 \times 20$ pixel areas in both stratum radiatum (dendrites) and stratum pyramidale (cell bodies) regions. Image intensities from stimulated regions $(200-250 \mu \mathrm{m}$ from the location of the stimulating electrode) were subtracted from unstimulated regions to give the difference in immunoreactivity. On rare occasion, the entire CA1 region was stained, and in these cases, the control region was measured in the CA3 area.

\section{RESULTS}

\section{Correlation with postsynaptic action potentials}

Staining for phospho-MAPK in sections from hippocampal slices was predicted to increase when stimulation typically effective in inducing LTP was delivered, because previous studies using immunoblots had shown increased phosphorylation of MAPK with this stimulation (English and Sweatt, 1996; Liu et al., 1999). Despite the significance of back-propagating dendritic action potentials to LTP (for review, see Linden, 1999; Paulson and Sejnowski, 2000), it is unknown whether action potentials are important for the activation of MAPK. We first tested whether the propagation of evoked population spikes across the CA1 subfield was accompanied by the appearance of staining for phosphoMAPK over a similar spatial area. Indeed, as the stimulation intensity increased, the greater the area of staining. The extent of staining across the CA1 subfield was significantly correlated with stimulation intensity $(p<0.001$; Spearman's rank order correlation) (Fig. 1A). Similarly, an intensity that typically failed to evoke population spikes (but evoked field synaptic responses) (Fig. $1 B, 60 \mu A, \sim 500 \mu \mathrm{m}$ from the stimulating electrode) also failed to induce staining in the same regions (Fig. $1 A, 60 \mu A$ ). A "pathological" stimulation intensity range was not likely to be necessary for the staining, because the population spikes had not reached asymptotic levels at even $140 \mu \mathrm{A}$ at the mid-CA1 recording site (Fig. 1B, bottom panel). We next tested whether blocking postsynaptic action potential spike generation and propagation with muscimol $(10 \mu \mathrm{M})$, an agonist of the GABA-A receptors, blocked the staining for phospho-MAPK. Muscimol was found to completely block the staining increase across CA1 in slices stimulated with TBS at $140 \mu \mathrm{A}(n=6$ slices; data not shown). Finally, the extent of staining induced with $140 \mu \mathrm{A}$ of TBS stimulation was severely curtailed by $20 \mu \mathrm{M}$ CNQX $(124 \pm 25.2 \mu \mathrm{m} ; n=3$ slices; data not shown), demonstrating that excitatory synaptic activity, and not electrical current per se, is responsible for the increase in phospho-MAPK staining.

\section{Dependence on frequency of stimulation}

With a stimulation intensity of $140 \mu \mathrm{A}$, TBS, as well as $100 \mathrm{~Hz}$ stimulation, induced intense staining for phospho-MAPK in hippocampal CA1. In many slices, distinct staining of the apical dendrites and cell bodies of CA1 pyramidal neurons could be identified (Fig. 2), although, in many instances, such structure was less obvious. This near-black staining is in stark contrast to the visible row of very lightly stained pyramidal cells in unstimulated slices, most evident at higher antibody concentrations (data not shown; but see Fig. $1 A$, top), indicating that the enzyme is maintained in a primarily unphosphorylated form in these neurons under control conditions.

In an effort to determine whether MAPK was activated under conditions not typically associated with LTP, we delivered the same number of pulses (120) at 1, 3, 5, and $10 \mathrm{~Hz}$. As shown in Figure 2, stimulation given at 5 and $10 \mathrm{~Hz}$ was as effective as stimulation at LTP-inducing frequencies in both stratum radia- 


\section{A Extent of PMAPK staining across CA1}
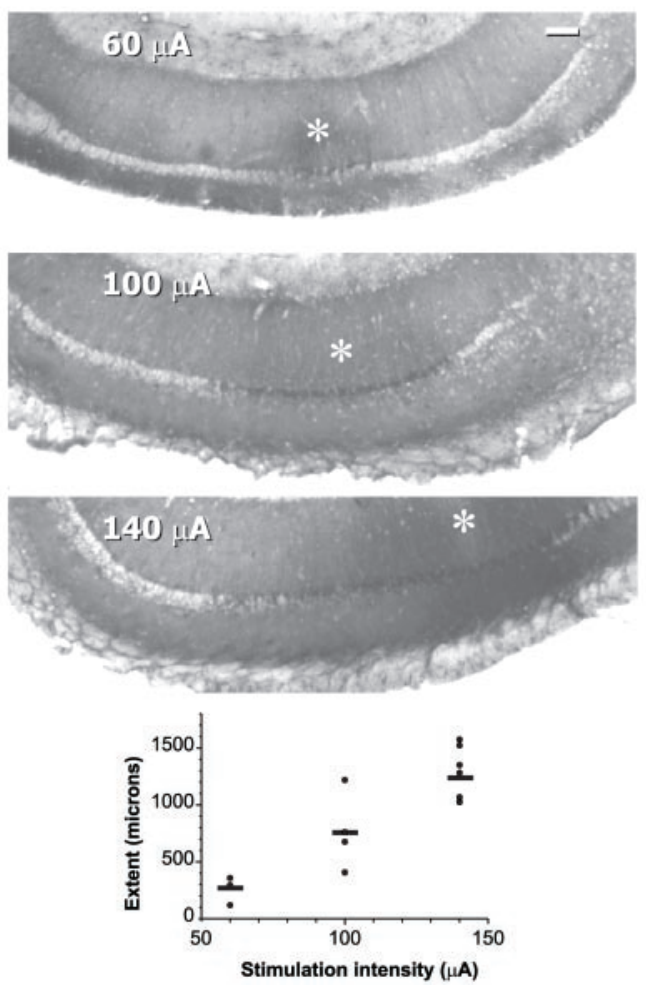

B Propagation of population spikes across CA1
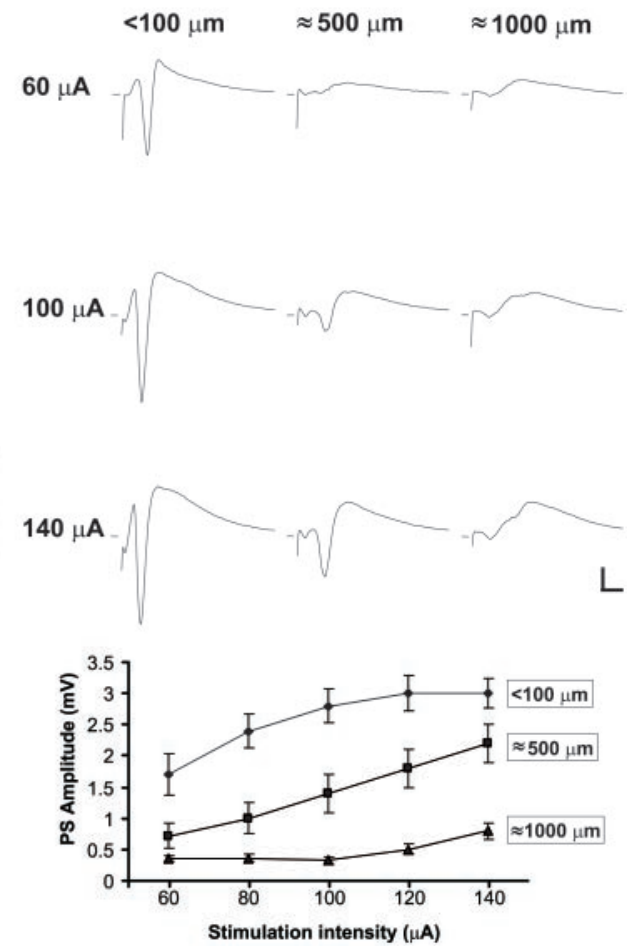

Figure 1. Extent of phospho-MAPK staining is related to the spread of population spikes across CA1. A, Staining for phospho-MAPK across CA1 was induced by theta-burst stimulation at three intensities, 60,100 , and $140 \mu \mathrm{A}$, and the largest distance between positive neuronal somata measured across CA1 (plotted at bottom left). The correlation between extent of staining and stimulation intensity was significant $(p<0.001$; Spearman's rank order correlation; $n=4,4$, and 6 slices, for 60,100 , and $140 \mu \mathrm{A}$, respectively). Scale bar, $100 \mu \mathrm{m}$. B, Spread of population spikes across CA1 depends on intensities similar to those for inducing phospho-MAPK staining. At $60 \mu \mathrm{A}$, little spread beyond the immediate vicinity of the stimulating electrode was observed (top row of traces). With stimulation intensities of 100 and $140 \mu \mathrm{A}$, visible population spikes were observed $500 \mu \mathrm{m}$ away (second column of traces). At $1 \mathrm{~mm}$ away from the stimulating electrode, population spikes were only discernable at and above $140 \mu \mathrm{A}$ (third column of traces). Portions of the stimulus artifacts were removed for clarity of the figure. Calibration: $0.6 \mathrm{mV}, 2.5 \mathrm{msec}$. Summary of population spike data are shown at the bottom $(n=7-9)$.

tum and stratum pyramidale regions. The increase in staining for phospho-MAPK was dependent, however, on stimulation frequency in that it was not activated with $1 \mathrm{~Hz}$ stimulation and was variably activated at $3 \mathrm{~Hz}$ (Fig. 2). The staining in response to 3 $\mathrm{Hz}$ occasionally reached levels of that at higher frequencies in individual cells but more often appeared in a lighter, perinuclear pattern and frequently failed to stain. At $1 \mathrm{~Hz}$, only the neurons appearing in direct contact with the stimulating electrode, some apparent interneurons, and unidentified fibers were stained. These results demonstrate that, when controlled for intensity of stimulation and number of pulses, frequency is a determining factor in the activation of MAP kinase and that stimulation at 5 $\mathrm{Hz}$ results in MAPK phosphorylation, although LTP is not typically induced by this frequency in our preparation.

\section{Dependence on route of calcium entry}

Different patterns of gene expression induced with neuronal activity have been suggested to rely on the spatial selectivity of the distribution of NMDA receptors and VSCCs on dendrites and somas, respectively, for selective regulation of genes (Ginty, 1997; Hardingham et al., 1999). Those studies, however, had been performed on neuronal and non-neuronal cell cultures in which the precise spatial stimulation of postsynaptic neurons was not usually possible. Accordingly, we pursued the question of whether NMDA- and VSCC-dependent calcium increases could be re- solved with the activation of MAPK using two different stimulation protocols (5 Hz and TBS). Interestingly, we found that $50 \mu \mathrm{M}$ D-APV, an NMDA-receptor antagonist, was effective in blocking the effects of $5 \mathrm{~Hz}$ stimulation but appeared ineffective against the TBS-induced phospho-MAPK staining (Fig. 3). In contrast, we found that $20 \mu \mathrm{M}$ nifedipine, which blocks L-type calcium channels, was ineffective in preventing phosphorylation of MAPK in stratum radiatum and pyramidale using either stimulation paradigm (Fig. 3). To get a significant blockade of the phosphoMAPK staining induced with TBS, a combination of APV and nifedipine was required (Fig. 3). APV and nifedipine together produced a slight additional decrease in the staining induced with $5 \mathrm{~Hz}$. As was the case with the different frequencies, the staining in stratum pyramidale paralleled that observed in stratum radiatum, with no apparent selectivity for the dendritic or somatic fields of CA1. These data show that, although different stimulation protocols can differentially recruit voltage-dependent calcium channels, any spatial selectivity between the two sources of calcium was not apparent with staining for phospho-MAPK.

\section{DISCUSSION}

Using the phosphorylation of MAPK, the experiments described here were designed to test the degree to which spatially distinct sources of calcium and frequency of stimulation can modulate 

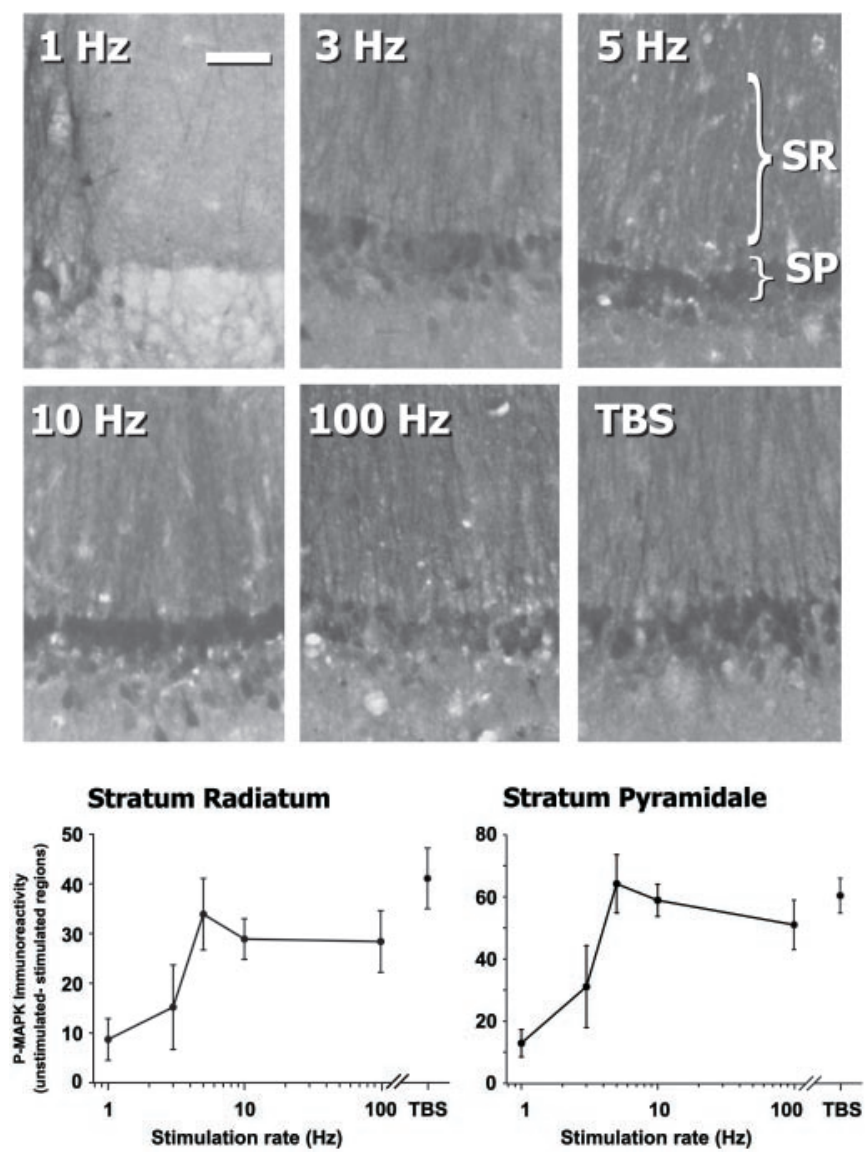

Figure 2. Staining for phospho-MAPK is frequency-dependent. Slices were stimulated for 120 pulses at $140 \mu \mathrm{A}$ at the indicated frequencies. In all cases, positive staining in stratum radiatum (dendrites) paralleled staining in stratum pyramidale (somata). Stained neurons on the left of the $1 \mathrm{~Hz}$ panel are cells near or contacting the stimulating electrode. Scale bar, $50 \mu \mathrm{m} . n=6,6,6,8,8$, and 7 slices for $1,3,5,10$, and $100 \mathrm{~Hz}$ and TBS, respectively.

cellular signaling in somatodendritic compartments. We found that the staining across CA1 for phospho-MAPK required stimulation intensities that approximate the recruitment of postsynaptic action potentials. Supporting this assertion is that the extent of the staining across CA1 could be severely reduced by inhibiting excitatory synaptic transmission with CNQX and completely blocked by a GABA-A agonist, muscimol, which decreased postsynaptic spiking and voltage-dependent currents. We also found that, although staining induced with different frequencies differ in their sensitivity to NMDA and VSCC antagonists, the spatial distribution of the staining was distinguished by neither frequency nor drug condition. Because under all pharmacological and physiological conditions tested dendritic staining correlated with somatic staining, these data are most consistent with the idea of cell-wide increases in calcium, or other second messengers, leading to the activation of MAPK in a cell-wide manner.

Our pharmacological studies have yielded several unexpected results. First, we found that, although both $5 \mathrm{~Hz}$ and TBS can induce the phosphorylation of MAPK, only TBS recruits VSCCs to a degree that seems capable of substituting for NMDA receptor activation in inducing the phospho-MAPK stain. These findings show that calcium, through VSCCs, which may be necessary for transcription of some genes, is differentially recruited with theta-burst stimulation but not with $5 \mathrm{~Hz}$ stimulation to produce
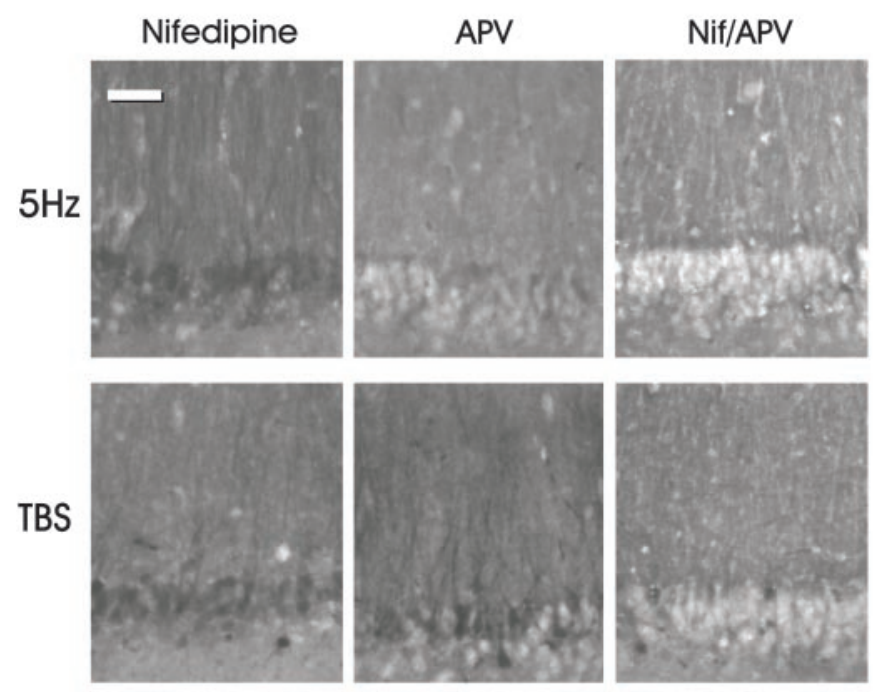

Stratum Radiatum

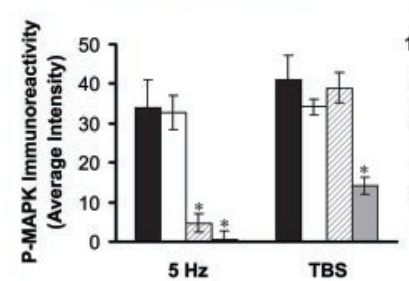

Stratum Pyramidale

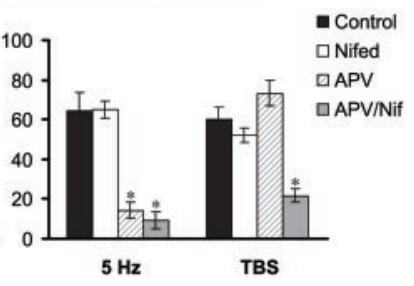

Figure 3. Phospho-MAPK staining induced with different stimulation displays distinct sensitivities to pharmacological blockade of calcium influx. Nifedipine did not reduce phospho-MAPK staining induced with either $5 \mathrm{~Hz}$ or theta-burst stimulation ( $n=7$ and 8 slices). APV significantly blocked staining induced with $5 \mathrm{~Hz}$ stimulation but not with TBS $(n=7$ and 6 slices). When TBS was used for stimulation, significant reduction of phospho-MAPK staining was only observed when NMDA receptors and VSCCs were both blocked $(n=9$ slices for TBS, 6 slices for $5 \mathrm{~Hz}$ ). Staining in stratum radiatum paralleled staining in stratum pyramidale under all pharmacological conditions.

increases in phospho-MAPK staining. Because MAPK itself seems to be activated under both conditions, however, other kinases and/or messengers may be responsible for distinguishing pulses (activation of NMDA receptors) from bursts (NMDA receptors and VSCCs) to generate different immediate-early gene responses. Activation of calcium-calmodulin-dependent protein kinases, shown to recruit CREB-binding protein, are good candidates for such a role (Chawla et al., 1998; Hu et al., 1999).

A second unexpected finding was that phosphorylation of MAPK in the soma appeared to parallel phosphorylation in the dendrites. We find this especially intriguing in the case of APV blockade of NMDA receptors in which one might have expected a soma-only staining pattern. This was not the case, because APV blocked both dendritic and somatic staining when $5 \mathrm{~Hz}$ stimulation was used, indicating that NMDA receptor activation was sufficient to lead to staining in the cell bodies. Also unexpected was that neither dendrite nor soma compartments were reduced with APV when theta-burst stimulation was used, indicating that VSCC are recruited to a degree that induces staining, even in the dendrites. Greater intensities of stimulation induce larger synaptic responses and resulting action potentials because they recruit a larger number of afferent fibers converging on postsynaptic neurons. Thus, although it is certainly true that higher intensities would recruit a spatially larger afferent pool and synaptic activation, it is not likely to explain the observation that staining of 
dendrites was not found without somatic staining. Usually, the dissociation tended to be the reverse; somatic staining in the absence of visible dendritic staining was sometimes observed at the margins of the stained areas, with the staining gradually decreasing in the most distal dendrites first. Our observations suggest that MAPK is not activated substantially in spatially discrete regions of the dendritic tree, but that it could instead be activated in a coordinated manner, cell-wide. This idea is consistent with our experiments showing a correlation of staining with the recruitment of action potentials and that action potentials can back-propagate into the apical dendritic tree (for review, see Linden, 1999).

Previous studies have investigated the activation of MAPK induced with electrical stimulation, but the range of control stimulus frequencies and intensities was limited [for example, 0.05 $\mathrm{Hz}$ (Impey et al., 1998) or 20 pulses at $20 \mathrm{~Hz}$ (Liu et al., 2000)]. The present findings are most compatible with the recent results of Winder et al. (1999), which correlated MAPK activation and LTP induction with stimulation that induces complex spikes (bursts of action potentials in CA1 neurons) (for review, see Lisman, 1997). Complex spikes evolved during $5 \mathrm{~Hz}$ stimulation and were facilitated by $\beta$-adrenergic receptor activation, which leads to the activation of PKA and MAPK-dependent signaling pathways (see also Thomas et al., 1998). Interestingly, both our study and that of Winder et al. (1999) showed that MAPK is activated by stimulus patterns that are not effective in inducing LTP, indicating that MAPK activation is not sufficient for LTP induction. Our present findings have extended the previous studies to indicate that somatic action potentials lead to the activation of MAPK via calcium influx requiring activation of L-type calcium channels and/or NMDA receptors.

In conclusion, different stimulus patterns lead to the activation of MAPK via different modes of calcium influx that are localized to different subcellular regions of the neuron. Although MAPK can be activated by several membrane receptors and types of neuronal excitation and can regulate a number of cellular substrates involved in synaptic plasticity, calcium influx through L-type calcium channels in response to somatic action potentials is most closely correlated with activation of MAPK in association with LTP.

\section{REFERENCES}

Atkins CM, Selcher JC, Petratis JJ, Trzaskos JM, Sweat JD (1998) The MAPK cascade is required for mammalian associative learning. Nat Neurosci 1:602-609.

Bading H, Greenberg ME (1991) Stimultion of protein tyrosine phosphorylation by NMDA receptor activation. Science 253:912-914.

Bading H, Ginty DD, Greenberg ME (1993) Regulation of gene expression in hippocampal neurons by distinct calcium signaling pathways. Science 260:181-186.

Baron C, Benes C, Tan HV, Fagard R, Roisin M-P (1996) Potassium chloride pulse enhances mitogen-activated protein kinase activity in rat hippocampal slices. J Neurochem 66:1005-1010.

Bliss TV, Lomo T (1973) Long-lasting potentiation of synaptic transmission in the dentate area of the anaesthetized rabbit following stimulation of the perforant path. J Physiol (Lond) 232:331-356.

Blum S, Moore AN, Adams F, Dash PK (1999) A mitogen-activated protein kinase cascade in the CA1/CA2 subfield of the dorsal hippocampus is essential for long-term spatial memory. J Neurosci 19:3535-3544.

Chawla S, Hardingham GE, Quinn DR, Bading H (1998) CBP: a signal- regulated transcriptional coactivator controlled by nuclear calcium and CaMK IV. Science 281:1505-1509.

Cummings JA, Mulkey RM, Nicoll RA, Malenka RC (1996) $\mathrm{Ca}^{2+}$ signaling requirements for long-term depression in the hippocampus. Neuron 16:825-833.

Davis S, Vanhoutte P, Pages C, Caboche J, Laroche S (2000) The MAPK/ERK cascade targets both Elk-1 and cAMP response elementbinding protein to control long-term potentiation-dependent gene expression in the dentate gyrus in vivo. J Neurosci 20:4563-4572.

Dudek SM, Bear MF (1992) Homosynaptic long-term depression in area CA1 of hippocampus and effects of $N$-methyl-D-aspartate receptor blockade. Proc Natl Acad Sci USA 89:4363-4367.

English JD, Sweatt JD (1996) Activation of p42 mitogen-activated protein kinase in hippocampal long-term potentiation. J Biol Chem 271:24329-24332.

English JD, Sweatt JD (1997) A requirement for the mitogen-activated protein kinase in hippocampal long-term potentiation. J Biol Chem 272:19103-19106.

Fiore RS, Bayer VE, Pelech SL, Posada J, Cooper JA, Baraban JM. (1993) p42 Mitogen-activated protein kinase in brain: prominent localization in neuronal cell bodies and dendrites. Neuroscience 55:463-472.

Ginty DD (1997) Calcium regulation of gene expression: isn't that spatial? Neuron 18:183-186.

Hardingham GE, Chawla S, Cruzalegui FH, Bading H (1999) Control of recruitment and transcription-activating function of CBP determines gene regulation by NMDA receptors and L-type calcium channels. Neuron 22:789-798

Hu SC, Chrivia J, Ghosh A (1999) Regulation of CBP-mediated transcription by neuronal calcium signaling. Neuron 22:799-808.

Impey S, Obrietan K, Wong ST, Poser S, Yano S, Wayman G, Deloulme JC, Storm DR (1998) Cross talk between ERK and PKA is required for $\mathrm{Ca}^{2+}$ stimulation of CREB-dependent transcription and ERK nuclear translocation. Neuron 21:869-883.

Impey S, Obrietan K, Storm DR (1999) Making new connections: role of ERK/MAP kinase signaling in neuronal plasticity. Neuron 23:11-14.

Kanterewicz BK, Urban NN, McMahon BT, Norman ED, Giffen LJ, Favata MF, Scherle PA, Trzaskos JM, Barrionuevo G, Klann E (2000) The extracellular signal-regulated kinase cascade is required for NMDA receptor-independent LTP in area CA1 but not CA3 of the hippocampus. J Neurosci 20:3057-3066.

Kurino M, Fukunaga K, Ushio Y, Miyamoto E (1995) Activation of mitogen-activated protein kinase in cultured rat hippocampal neurons by stimulation of glutamate receptors. J Neurochem 65:1282-1289.

Larson J, Wong D, Lynch G (1986) Patterned stimulation at the theta frequency is optimal for the induction of hippocampal long-term potentiation. Brain Res 368:347-350.

Linden DJ (1999) The return of the spike: postsynaptic action potentials and the induction of LTP and LTD. Neuron 22:661-666.

Lisman JE (1997) Bursts as a unit of neural information: making unreliable synapses reliable. Trends Neurosci 20:38-43.

Liu J, Fukunaga K, Yamamoto H, Nishi K, Miyamoto E (1999) Differential roles of $\mathrm{Ca}^{2+} /$ calmodulin-dependent protein kinase II and mitogen-activated protein kinase activation in hippocampal long-term potentiation. J Neurosci 19:8292-8299.

Nakazawa J, Murphy TH (1999) Activation of nuclear calcium dynamics by synaptic stimulation in cultured cortical neurons. J Neurochem 73:1075-1083.

Paulsen O, Sejnowski TJ (2000) Natural patterns of activity and longterm synaptic plasticity. Curr Opin Neurobiol 10:172-179.

Quinlan EM, Halpain S (1996) Emergence of activity-dependent, bidirectional control of microtubule-associated protein MAP2 phosphorylation during postnatal development. J Neurosci 16:7627-7637.

Rosen LB, Ginty DD, Weber MJ, Greenberg ME (1994) Membrane depolarization and calcium influx stimulate MEK and MAP kinase via activation of Ras. Neuron 12:1207-1221.

Thomas MJ, Watabe AM, Moody TD, Makhinson M, O’Dell TJ (1998) Postsynaptic complex spike bursting enables the induction of LTP by theta frequency synaptic stimulation. J Neurosci 18:7118-7126.

Winder DG, Martin KC, Muzzio IA, Roher D, Chruscinski A, Kobilka B, Kandel ER (1999) ERK plays a regulatory role in induction of LTP by theta frequency stimulation and its modulation by $\beta$-adrenergic receptors. Neuron 24:715-726.

Xia Z, Dudek H, Miranti CK, Greenberg ME (1996) Calcium influx via the NMDA receptor induces immediate early gene transcription by a MAP kinase/ERK-dependent mechanism. J Neurosci 16:5425-5436. 